\title{
The Kink Casimir Energy in a Lattice Sine-Gordon Model
}

\author{
J M Speight* \\ Department of Mathematical Sciences, \\ University of Durham, Durham DH1 3LE
}

\begin{abstract}
The Casimir energy of quantum fluctuations about the classical kink configuration is computed numerically in the weak coupling approximation for a recently proposed lattice sineGordon model. This energy depends periodically on the kink position and is found to be approximately sinusoidal.

PACS classification numbers: 03.65.Sq, 11.10.Lm, 63.10.+a.
\end{abstract}

\section{Introduction}

Classically, a topologically stable field configuration may be regarded as lying in a potential well in an infinite dimensional configuration space. Two solutions in different sectors are separated by an infinite potential barrier by virtue of the topology. Quantum mechanically, a particle cannot sit at the bottom of a well: it always possesses a zero point energy dependent on the shape of the well bottom. The analogous situation applies to fields also.

We wish to find the zero point energy associated with a kink configuration in a certain lattice sine-Gordon model. The model was chosen because it has an exact lattice version of the topological lower bound on kink energy, and an explicit static kink which saturates this bound. The calculation is performed in the weak coupling approximation, by performing a Taylor expansion of the potential about the kink configuration, truncating the expansion at quadratic order. We then find the normal modes of the truncated system, reducing the problem to that of an infinite set of decoupled harmonic oscillators. On quantization, each contributes a zero point energy. Summing over all oscillators gives an infinite total.

If we similarly approximate the vacuum well-bottom we can derive the zero point energy associated with the trivial vacuum. This is also infinite. The "physical" quantity required is the energy difference between the kink and the vacuum, since we can always define the vacuum energy to be zero. This quantity, analogous to the Casimir energy of Quantum Electrodynamics [1], turns out to be finite and less than the classical energy of the kink. Since the model possesses only discrete translation symmetry, the normal mode frequencies, and hence the Casimir energy depend periodically on the kink position.

Calculation of the normal mode frequencies amounts to finding the eigenvalues of an infiniteorder, tridiagonal, symmetric matrix. In practice this is not possible and the system must be truncated symmetrically about the kink centre, ignoring the large $|x|$ degrees of freedom. It is possible to show that the resulting quantum energy correction must be negative, for any size of truncated system. The correction may be calculated numerically.

*Present address: Department of Mathematics, University of Texas at Austin, Austin TX 78712, USA 


\section{The Lattice Sine-Gordon Model}

Consider the lattice sine-Gordon model (LSGM) defined by the Lagrangian [2],

$$
L=\frac{h}{4} \sum_{j \in \mathcal{Z}}\left[\dot{\psi}_{j}^{2}-\frac{4}{\lambda^{2} h^{2}} \sin ^{2} \frac{\lambda}{2}\left(\psi_{j+1}-\psi_{j}\right)-\frac{m^{2}}{\lambda^{2}} \sin ^{2} \frac{\lambda}{2}\left(\psi_{j+1}+\psi_{j}\right)\right],
$$

where $h$ is the lattice spacing, $m$ is a mass parameter, $\lambda$ is a dimensionless coupling constant and $\mathcal{Z}$ denotes the set of integers. Taking the $h \rightarrow 0$ limit one recovers the standard sine-Gordon model:

$$
\lim _{h \rightarrow 0} L=\frac{1}{4} \int_{-\infty}^{\infty} d x\left[\left(\frac{\partial \psi}{\partial t}\right)^{2}-\left(\frac{\partial \psi}{\partial x}\right)^{2}-\frac{m^{2}}{\lambda^{2}} \sin ^{2} \lambda \psi\right],
$$

where $x=h j$. The standard sine-Gordon Lagrangian is more usually written in terms of $\phi=2 \psi$ and differs by a factor of 8 , but the above form is more convenient.

The LSGM has an infinite set of discrete potential minima,

$$
\psi=\frac{n \pi}{\lambda},
$$

where $n \in \mathcal{Z}$, and a static kink interpolating between neighbouring minima, which may be derived using a Bogomol'nyi argument. The energy of a static configuration is just the potential $V$, and

$$
\begin{aligned}
0 & \leq \frac{h}{4} \sum_{j}\left(\frac{2}{\lambda h} \sin \frac{\lambda}{2}\left(\psi_{j+1}-\psi_{j}\right)-\frac{m}{\lambda} \sin \frac{\lambda}{2}\left(\psi_{j+1}+\psi_{j}\right)\right)^{2} \\
& =V+\frac{m}{2 \lambda^{2}} \sum_{j}\left(\cos \lambda \psi_{j+1}-\cos \lambda \psi_{j}\right) \\
\Rightarrow V & \geq \frac{m}{\lambda^{2}}
\end{aligned}
$$

when kink boundary conditions are imposed. This (the Bogomol'nyi) bound is saturated if and only if

$$
\sin \frac{\lambda}{2}\left(\psi_{j+1}-\psi_{j}\right)=h m \sin \frac{\lambda}{2}\left(\psi_{j+1}+\psi_{j}\right) .
$$

The first order difference equation, (5), is called the Bogomol'nyi equation and, remarkably, has an explicit kink solution:

$$
\psi_{j}=\frac{2}{\lambda} \tan ^{-1}\left[\left(\frac{2+h m}{2-h m}\right)^{j-b / h}\right] .
$$

The dimensionless parameter $h m \in(0,2)$ for sensible solutions. The arbitrary constant $b$ may take any real value - the kink energy is not position dependent. The right hand side of (依) may be interpreted as the classical kink mass, $M$. Small velocity kink dynamics can then be approximated by geodesic motion of a point particle, mass $M$, on the manifold generated by $b$ translation, with a natural induced metric [2].

\section{The Weak Coupling Approximation}

We follow the method outlined in [3] adapted for the infinite lattice. We work in natural units, effectively having absorbed a factor $\sqrt{\hbar}$ into $\lambda$ so that, by the standard argument, the weak coupling criterion $\lambda \ll 1$ yields a semi-classical approximation. 
The momentum conjugate to $\psi_{j}$ is

$$
\pi_{j}=\frac{\partial L}{\partial \dot{\psi}_{j}}=\frac{h}{2} \dot{\psi_{j}}
$$

Thus, the LSGM Hamiltonian is

$$
H=\sum_{j} \frac{\pi_{j}^{2}}{h}+V(\psi)
$$

where

$$
V(\psi)=\frac{h}{4} \sum_{j}\left[\frac{4}{\lambda^{2} h^{2}} \sin ^{2} \frac{\lambda}{2}\left(\psi_{j+1}-\psi_{j}\right)+\frac{m^{2}}{\lambda^{2}} \sin ^{2} \frac{\lambda}{2}\left(\psi_{j+1}+\psi_{j}\right)\right]
$$

Let $\tilde{\psi}$ be a static configuration giving a local (in configuration space) minimum of the potential, $V(\psi)$. We treat motion about this stable configuration in the small $\lambda$ approximation by Taylor expansion of $V$ :

$$
V(\psi)=V(\tilde{\psi})+\frac{m}{2} \sum_{j, k} W_{j k}\left(\psi_{j}-\tilde{\psi}_{j}\right)\left(\psi_{k}-\tilde{\psi}_{k}\right)+\cdots
$$

where 


$$
\begin{aligned}
W_{j k}= & \left.\frac{1}{m} \frac{\partial^{2} V}{\partial \psi_{j} \partial \psi_{k}}\right|_{\tilde{\psi}} \\
= & \frac{h m}{4}\left(\delta _ { j , k } \left\{\frac{2}{(h m)^{2}}\left[\cos \lambda\left(\tilde{\psi}_{k}-\tilde{\psi}_{k-1}\right)+\cos \lambda\left(\tilde{\psi}_{k+1}-\tilde{\psi}_{k}\right)\right]\right.\right. \\
& \left.+\frac{1}{2}\left[\cos \lambda\left(\tilde{\psi}_{k}+\tilde{\psi}_{k-1}\right)+\cos \lambda\left(\tilde{\psi}_{k+1}+\tilde{\psi}_{k}\right)\right]\right\} \\
& +\delta_{j, k-1}\left[-\frac{2}{(h m)^{2}} \cos \lambda\left(\tilde{\psi}_{k}-\tilde{\psi}_{k-1}\right)+\frac{1}{2} \cos \lambda\left(\tilde{\psi}_{k}+\tilde{\psi}_{k-1}\right)\right] \\
& \left.+\delta_{j, k+1}\left[-\frac{2}{(h m)^{2}} \cos \lambda\left(\tilde{\psi}_{k+1}-\tilde{\psi}_{k}\right)+\frac{1}{2} \cos \lambda\left(\tilde{\psi}_{k+1}+\tilde{\psi}_{k}\right)\right]\right) .
\end{aligned}
$$

Note that $W$ is a real, symmetric, tridiagonal matrix. Note also that the next term in the expansion is

$$
\left.\frac{1}{3 !} \sum_{i, j, k} \frac{\partial^{3} V}{\partial \psi_{i} \partial \psi_{j} \partial \psi_{k}}\right|_{\tilde{\psi}}\left(\psi_{i}-\tilde{\psi}_{i}\right)\left(\psi_{j}-\tilde{\psi}_{j}\right)\left(\psi_{k}-\tilde{\psi}_{k}\right),
$$

and that the three derivatives with respect to $\psi$ introduce a factor of $\lambda^{3}$, leaving an overall factor of $\lambda$ after cancelling the $1 / \lambda^{2}$ of $V(\psi)$. Thus, higher corrections are at least of order $\lambda$, which is why one can truncate the series in the small $\lambda$ limit.

Owing to the symmetry of the $W$ matrix, there exists an orthogonal transformation $R$ such that

$$
W_{j k}=\sum_{l, m} R_{j l}^{T} U_{l m} R_{m k}
$$

where $U$ is a diagonal matrix. We may reduce the system to a sequence of decoupled harmonic oscillators by transforming to the rotated coordinates (normal coordinates)

$$
\xi_{j}=\sum_{k} R_{j k}\left(\psi_{k}-\tilde{\psi}_{k}\right),
$$

which have conjugate momenta

$$
\eta_{j}=\sum_{k} R_{j k} \pi_{k}
$$

Then,

$$
\frac{h}{2}(H-V(\tilde{\psi}))=\frac{1}{2} \sum_{j}\left(\eta_{j}^{2}+\frac{h m}{2} \Omega_{j}^{2} \xi_{j}^{2}\right)
$$

where $\Omega_{j}^{2}$ are the eigenvalues of the $W$ matrix, none of which can be negative since $\tilde{\psi}$ locally minimizes the potential.

We now quantize in standard canonical fashion, taking $\tilde{\psi}$ to be first the vacuum, then the kink located at $x=b$. The vacuum ground state energy is

$$
E_{\{0\}}^{0}=\frac{m}{\sqrt{2 h m}} \sum_{j} \Omega_{j}^{0} .
$$

The kink, by virtue of the zero mode $b$ of (6) lies not in a potential well, but in a level-bottomed valley meandering through configuration space. One of the normal modes is locally tangential to the valley bottom and consequently has vanishing corresponding eigenvalue (zero frequency). We shall treat this translation mode, $b$, classically because in the weak coupling approximation it is much heavier than the orthogonal modes (mass $m / \lambda^{2}$ compared with $m$ ). 
While the translation orbit of the static kink (6) is an equipotential curve, neighbouring orbits are not: the potential varies periodically along them. So the eigenvalues of the $W$ matrix $\left(\Omega_{j}^{K}\right)^{2}$ will be $b$ dependent with period $h$. The ground state energy of a kink at $b$ is

$$
E_{\{0\}}^{K}(b)=\frac{m}{\lambda^{2}}+\frac{m}{\sqrt{2 h m}} \sum_{j} \Omega_{j}^{K}(b) .
$$

where the sum may be taken over all eigenvalues since the one we wish to omit is zero anyway.

There is no reason to expect either $E_{\{0\}}^{K}(b)$ or $E_{\{0\}}^{0}$ to converge to a finite sum but one would expect finite Casimir energy

$$
\mathcal{E}=E_{\{0\}}^{K}(b)-E_{\{0\}}^{0}-\frac{m}{\lambda^{2}}
$$

since the lattice spacing $h$ has effectively cut off the ultra violet divergence problems of the continuum model. We must still take care when manipulating the divergent sums of (19) that they are suitably regulated before being combined. The method of regulation is determined by practical considerations: we calculate $E_{\{0\}}^{K}(b)$ and $E_{\{0\}}^{0}$ on a finite lattice, compute $\mathcal{E}$ and then allow the lattice size to grow large. In practice we must truncate the lattice to finite size anyway in order to solve the kink matrix eigenvalue problem.

\section{The Eigenvalue Problem}

We now address the problem of finding the eigenvalues of the vacuum and kink $W$ matrices, $W^{0}$ and $W^{K}$. Let us first consider the vacuum matrix, obtained from (11) by substituting $\tilde{\psi}_{k}=0$ :

$$
W_{j k}^{0}=\frac{h m}{4}\left[\left(\frac{4}{(h m)^{2}}+1\right) \delta_{j, k}-\left(\frac{2}{(h m)^{2}}-\frac{1}{2}\right)\left(\delta_{j, k-1}+\delta_{j, k+1}\right)\right] .
$$

This is a very simple matrix. All the diagonal entries are equal, as are all the upper and lower diagonal entries. Consequently, the spectrum of the truncated matrix of order $N$ is known exactly (see, for example, 㧅):

$$
\left(\Omega_{j}^{0}\right)^{2}=\frac{4+h^{2} m^{2}}{4 h m}-\frac{4-h^{2} m^{2}}{4 h m} \cos \left(\frac{j \pi}{N+1}\right),
$$

$j=1,2, \ldots, N$. In the $N \rightarrow \infty$ limit this is strikingly similar to the dispersion relation for phonons on the lattice [2] because the normal modes of oscillation about the vacuum are phonons.

The question of making sense of the $N \rightarrow \infty$ limit of $\sum_{j} \Omega_{j}^{0}$ is irrelevant because there is no hope of finding the exact spectrum of the kink $W$ matrix, $W^{K}$. Substituting (6) into (11) we obtain an explicit expression for $W^{K}(b)$. Since the kink is highly localized (provided $h m$ is not too small), at large $|j|$ the kink configuration rapidly approaches neighbouring vacuum minima so that $W_{j k}^{K}(b)$ tend to $W_{j k}^{0}$ away from the matrix centre. The suggestion, then, is that we truncate the lattice, pinning all the large $|j|$ degrees of freedom, for which the kink and vacuum configurations are essentially identical, to their classical values. The resulting finite problem may be solved numerically.

One can prove that, whatever the order, $N$ (an odd integer), of the symmetrically truncated system (that is, truncated symmetrically about the lattice site $j=0$ ), the Casimir energy must be negative. The proof rests on the observation that, truncated to order $N=2 n+1$,

$$
W^{K}=W^{0}+B+C
$$


where $B$ is the symmetric, tridiagonal matrix,

$$
\begin{aligned}
& B=\left(\begin{array}{ccccccc}
-\beta_{-n} & b_{-n} & 0 & & & & \\
b_{-n} & -\beta_{-n+1} & b_{-n+1} & & & & \\
0 & b_{-n+1} & -\beta_{-n+2} & & & & \\
& & & \ddots & & & \\
& & & & -\beta_{n-2} & b_{n-1} & 0 \\
& & & & b_{n-1} & -\beta_{n-1} & b_{n} \\
& & & & 0 & b_{n} & -\beta_{n}
\end{array}\right), \\
& b_{j}=\frac{h m}{8}\left(1-\cos \lambda\left(\tilde{\psi}_{j}+\tilde{\psi}_{j-1}\right)\right)+\frac{1}{2 h m}\left(1-\cos \lambda\left(\tilde{\psi}_{j}-\tilde{\psi}_{j-1}\right)\right) \\
& \geq 0, \\
& \beta_{j}=b_{j}+b_{j+1},
\end{aligned}
$$

and $C$ is the diagonal matrix with elements,

$$
C_{i j}=\delta_{i j} \frac{h m}{4}\left[\cos \lambda\left(\tilde{\psi}_{j+1}+\tilde{\psi}_{j}\right)+\cos \lambda\left(\tilde{\psi}_{j}+\tilde{\psi}_{j-1}\right)-2\right] .
$$

Now, $C$ manifestly has negative semi-definite eigenvalues, and $B$ is a special case of a class of matrices whose spectra are known to be negative semi-definite [5]. Regarding $B$ and $C$ as perturbations to the matrix $W^{0}$, we apply a corollary of the minimax theorem [6]:

Theorem: If $\Theta, \Upsilon, \Gamma$ are symmetric $N \times N$ matrices with eigenvalues $\theta_{r}, v_{s}, \gamma_{t}$ (all sets arranged in non-increasing order), and

$$
\Theta=\Upsilon+\Gamma
$$

then

$$
\theta_{r} \leq v_{r}+\gamma_{1}
$$

for all $r=1,2, \ldots, N$.

Thus the eigenvalues of $W^{0}+B$ are shifted down relative to those of $W^{0}$ (the greatest eigenvalue of $B$ is negative or zero), and similarly, the eigenvalues of $W^{0}+B+C=W^{K}$ are shifted down relative to those of $W^{0}+B$. Hence,

$$
\sum_{j} \Omega_{j}^{K}(b) \leq \sum_{j} \Omega_{j}^{0}
$$

and, substituting into 19,

$$
\mathcal{E}(b) \leq 0,
$$

the quantum mechanical effect must be to lower the kink energy.

We have assumed, on physical grounds, that

$$
\mathcal{E}_{N}(b)=\frac{m}{\sqrt{2 h m}} \sum_{j}\left(\Omega_{j}^{K}(b)-\Omega^{0}\right),
$$

the Casimir energy of the order $N$ truncated system converges to a constant value as $N$ grows large. Numerical evidence for this is presented in figure 1 , a graph of $N$ against $\mathcal{E}_{N}(0) / m$ for various values of $h m$. Convergence is very fast for large $h m$ (the $h m=1.9$ curve is essentially flat for $N \geq 3$ ) but much slower for small $\mathrm{hm}$. Of course, this happens because the more finely space is 
discretized, the more degrees of freedom the kink structure is spread over. There is also a much smaller exacerbating effect due to the $h m$ dependence in the kink solution, (6) - large $h m$ kinks are sharper in "real" $x$ space than small $h m$ kinks. Note that, as proved above, the Casimir energy is always negative and that its magnitude grows large for small $\mathrm{hm}$ as we expect from consideration of the unrenormalized continuum model $[7]$. Although $b=0$ was chosen for these data, the rates of convergence are virtually independent of $b \in[-h / 2, h / 2]$.

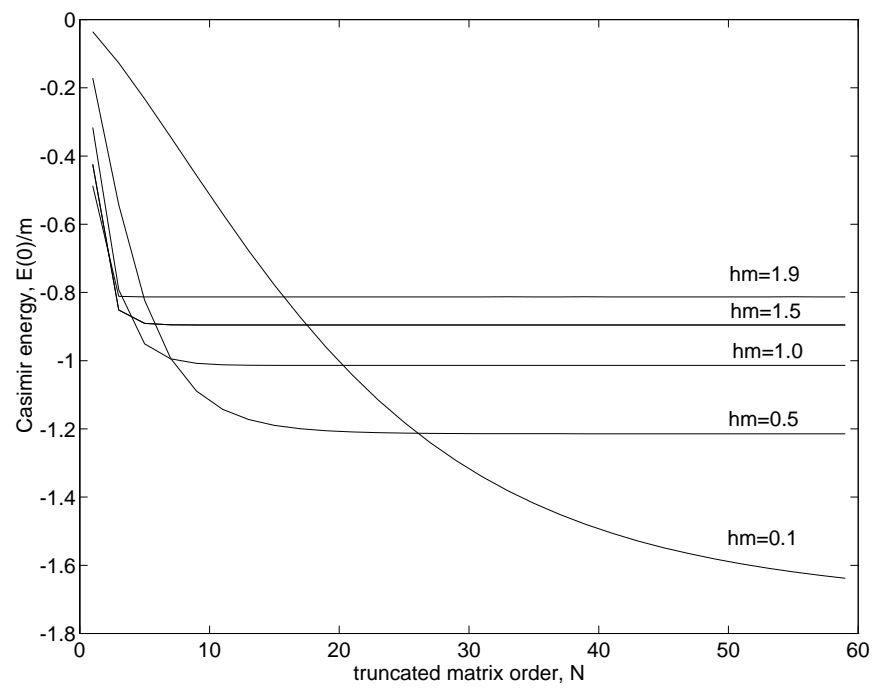

Figure 1: Convergence of the Casimir energy.

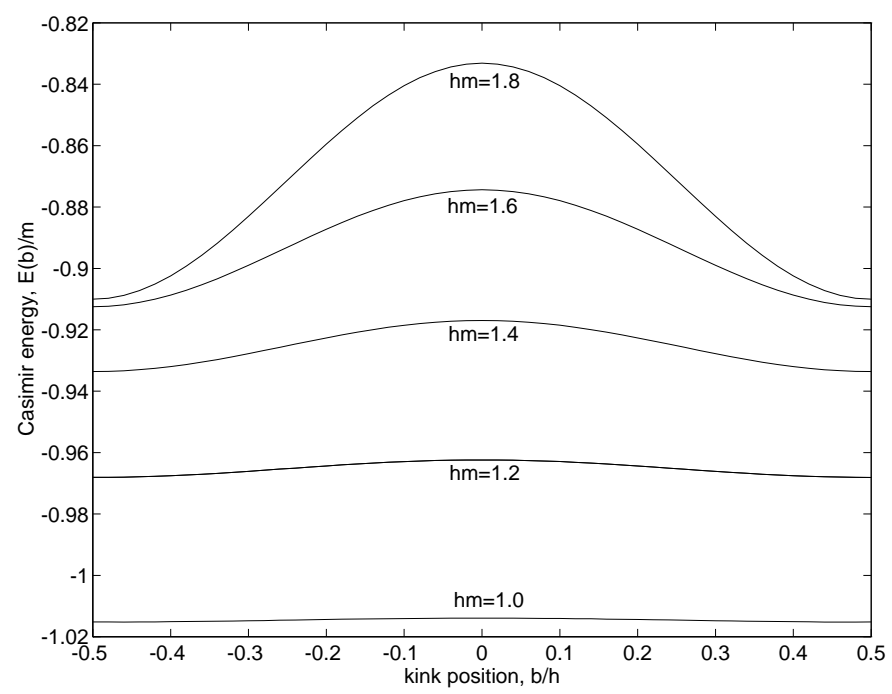

Figure 2: Position dependence of the Casimir energy.

The Casimir energy of suitably truncated systems is plotted as a function of kink position $b$ in figure 2. It attains its maxima where the kink is located exactly on a lattice site and its minima where the kink is halfway between two sites. For large $h m$ the resemblance to a sinusoidal curve is remarkable: figure 3 shows a sinusoidal fit for $h m=1.8$. The resemblence deteriorates as $h m$ 
decreases because numerical errors become relatively large as the Casimir energy barrier $[\mathcal{E}(0)-$ $\mathcal{E}(h / 2)] / m$ decreases (figure 4$)$. Striking though the similarity is, the author can find no analytic evidence to support the claim that $\mathcal{E}(b)$ really is sinusoidal.

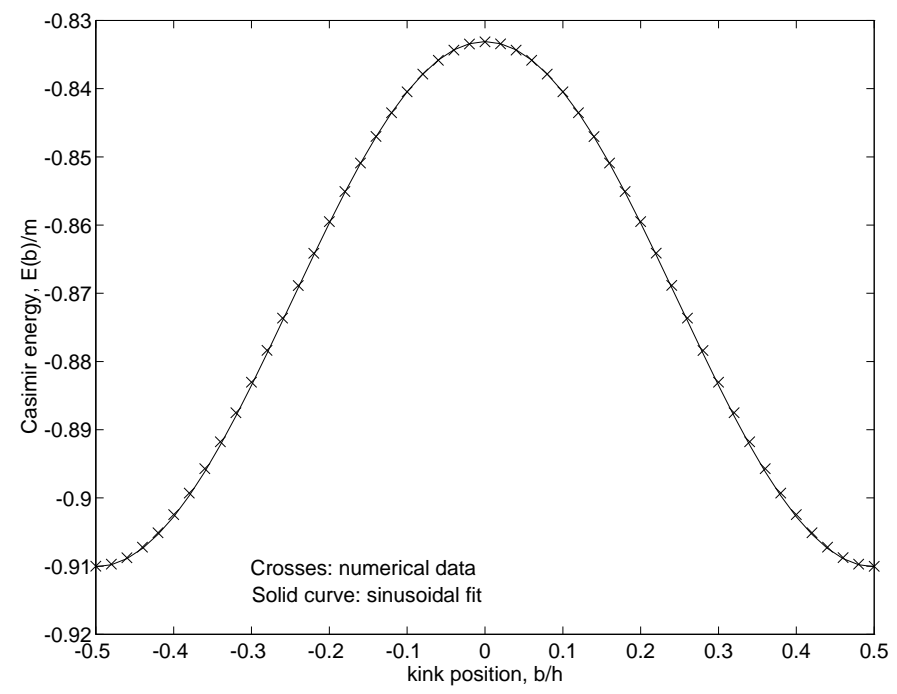

Figure 3: Sinusoidal fit to the $h m=1.8$ Casimir energy.

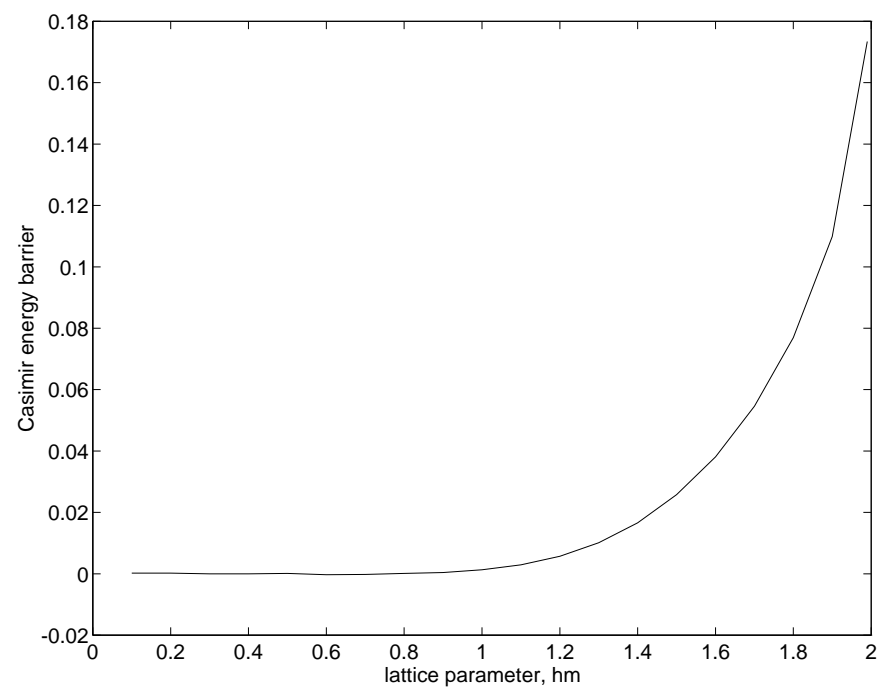

Figure 4: The effect of discreteness on the Casimir energy barrier.

It is the Casimir energy barrier (figure 4) which is physically most relevant and which will directly affect classical kink dynamics. In the continuum limit full continuous translation symmetry is recovered, so the barrier should disappear, as is indicated by the plot. In fact, in common with other (classical kinetic) discreteness effects of the LSGM [2], the barrier is very small for all $h m<1$. If $\mathcal{E}(b) / m$ for a given $b / h$ is a strictly increasing function of $h m \in(0,2)$, as it appears to be, then given that $\mathcal{E}(b)<0$, the barrier should approach a finite value in the $h m \rightarrow 2$ limit. Numerical evidence suggests that it does and that this value is around 0.17 . 


\section{Concluding Remarks}

We have seen that quantum fluctuations around the kink configuration spoil the level kink valley bottom by introducing a Casimir energy which depends periodically on the (classical) kink position. This energy has been computed numerically in the weak coupling approximation and found to be approximately sinusoidal, maximum when $b=0, \pm h, \pm 2 h, \ldots$ and minimum when $b= \pm h / 2, \pm 3 h / 2, \pm 5 h / 2, \ldots$, the difference between these extrema being large for large $h m$ but rapidly vanishing in the continuum limit. It is superficially similar to the Peierls-Nabarro potential of the Frenkel-Kontorova model [8] (the conventional lattice sine-Gordon model) but is entirely different in origin, being a purely quantum effect. Since the Casimir effect is a genuine physical phenomenon, experimentally verified in the context of Quantum Electrodynamics, we are led to the conclusion that classical kinks in this lattice model may be "pinned" by the quantum mechanics of the orthogonal modes.

Finally, following a suggestion by Gibbons and Manton [9] we could attempt to include the effect of variation of orthogonal mode frequencies on the quantized geodesic approximation of kink motion by including the Casimir energy as an extra potential term in the Hamiltonian:

$$
\widehat{H}_{G A}=-\frac{1}{2} \Delta+\mathcal{E}(b)
$$

where $\Delta$ is the covariant Laplacian on the submanifold of static kinks. Given the periodic nature of $\mathcal{E}$, we would then expect band structure in the kink spectrum. There are two objections to this. First, the kink kinetic terms are of order $\lambda^{2}$ (due to the large kink mass), while the potential was expanded only up to order $\lambda^{0}$, so the perturbative expansion is not consistent. Second, picking out the $-\frac{1}{2} \Delta$ kinetic term at order $\lambda^{2}$ is also not consistent because there are several other kinetic terms at this order neglected in equation (30). This may be seen by reëxpressing the kinetic Hamiltonian of the full quantum field theory,

$$
\widehat{T}=\frac{1}{h} \sum_{j} \frac{\partial^{2}}{\partial \psi_{j}^{2}},
$$

(an infinite dimensional Laplacian) in terms of the kink translation mode $b$ and the orthogonal modes $\xi_{j}$. The resulting formula is very messy, and includes order $\lambda^{2}$ cross terms with derivatives $\partial^{2} / \partial \xi_{j} \partial b$ and $\partial^{2} / \partial \xi_{j} \partial \xi_{k}$ in addition to the terms included in (30). The analogous expression in the continuum model [3] can be greatly simplified by boosting to the kink's rest frame, but this trick is not available to us here. These considerations cast doubt on the suggested procedure, at least in this case. However, one should note that a naive expansion of the Hamiltonian in $\lambda$ may not be the most physically relevant procedure when considering quantum kink dynamics. For example, one could imagine making the demand that the kink's "speed" (in an appropriate quantum sense) be of order $\lambda^{0}$. Given the kink's large mass in the weak coupling approximation, one would then expect the kink kinetic term to dominate the Hamiltonian. So the legitimacy of the suggested procedure remains an open and somewhat controversial question.

Acknowledgments: I would like to thank Bernd Schroers for many useful discussions and Richard Ward for advice and comments. I also acknowledge the financial support of the UK Science and Engineering Research Council in the form of a research studentship.

\section{References}

[1] Plunien G, Müller B and Greiner W, The Casimir Effect Phys. Rep. 134 (1986) 87.

[2] Speight J M and Ward R S, Kink Dynamics in a Novel Discrete Sine-Gordon System Nonlinearity 7 (1994) 475. 
[3] Rajaraman R, Solitons and Instantons North Holland 1982.

[4] Bellman R, Introduction to Matrix Analysis Tata McGraw Hill 1974.

[5] Ledermann W and Reuter G E, Spectral Theory for Differential Equations of Simple Birth and Death Processes Phil. Trans. Royal Soc. London ser A vol 246 (1953-1954) 321-369.

[6] Wilkinson J H, The Algebraic Eigenvalue Problem Oxford University Press 1965.

[7] Dashen R F, Hasslacher B and Neveu A, Particle Spectrum in Model Field Theories from Semiclassical Functional Integral Techniques Phys. Rev. D11 (1975) 3424.

[8] Peyrard M and Kruskal M D, Kink Dynamics in the Highly Discrete Sine-Gordon System Physica 14D (1984) 88.

[9] Gibbons G W and Manton N S, Classical and Quantum Dynamics of BPS Monopoles Nucl. Phys. B274 (1986) 183. 